\title{
Novel Behavioral Paradigm Reveals Lower Temporal Limits on Mouse Olfactory Decisions
}

\author{
Arbora Resulaj ${ }^{1,2}$ and Dmitry Rinberg ${ }^{1,3}$ \\ ${ }^{1}$ Janelia Farm Research Campus, Howard Hughes Medical Institute, Ashburn, Virginia 20147, ${ }^{2}$ Howard Hughes Medical Institute, Center for Neural Circuits \\ and Behavior, Neurobiology Section and Department of Neuroscience, University of California San Diego, La Jolla, California 92093, and ${ }^{3}$ New York \\ University Neuroscience Institute, New York University Langone Medical Center, New York, New York 10016
}

Temporal limits on perceptual decisions set strict boundaries on the possible underlying neural computations. How odor information is encoded in the olfactory system is still poorly understood. Here, we sought to define the limit on the speed of olfactory processing. To achieve this, we trained mice to discriminate different odor concentrations in a novel behavioral setup with precise odor delivery synchronized to the sniffing cycle. Mice reported their choice by moving a horizontal treadmill with their front limbs. We found that mice reported discriminations of $75 \%$ accuracy in 70-90 ms after odor inhalation. For a low concentration and nontrigeminal odorant, this time was $90-140 \mathrm{~ms}$, showing that mice process odor information rapidly even in the absence of trigeminal stimulation. These response times establish, after accounting for odor transduction and motor delays, that olfactory processing can take tens of milliseconds. This study puts a strong limit on the underlying neural computations and suggests that the action potentials forming the neural basis for these decisions are fired in a few tens of milliseconds.

\section{Significance Statement}

Understanding how sensory information is processed requires different approaches that span multiple levels of investigation from genes to neurons to behavior. Limits on behavioral performance constrain the possible neural mechanisms responsible for specific computations. Using a novel behavioral paradigm, we established that mice can make decisions about odor intensity surprisingly fast. After accounting for sensory and motor delays, the limit on some olfactory neural computations can be as low as a few tens of milliseconds, which suggests that only the first action potentials across a population of neurons contribute to these computations.

\section{Introduction}

Recently, the mouse has become a very powerful model organism for studying neural computations because it allows the combination of a variety of approaches at different levels from molecular and genetic to computational and behavioral. Despite great progress in mouse neurobiology, little is known about the temporal limits on mouse behavioral performance.

Olfaction, being presumably the most genetically tractable and anatomically compact sensory system, presents a great opportunity for testing the basic principles of sensory processing

\footnotetext{
Received Nov. 13, 2014; revised July 7, 2015; accepted July 17, 2015.

Author contributions: A.R. and D.R. designed research; A.R. performed research; A.R. and D.R. analyzed data; A.R. and D.R. wrote the paper.

This work was supported by the Howard Hughes Medical Institute. We thank J. Dudman, M. Smear, R. Shusterman, and A. Resulaj for helpful discussions; T. Tabachnik for help with designing the behavioral rig; and T. Bozza, R. Kiani, M. Smear, and C. Wilson for helpful comments on the manuscript.

The authors declare no competing financial interests.

Correspondence should be addressed to Dmitry Rinberg, New York University Neuroscience Institute, New York University Langone Medical Center, 450 East 29th Street, Room 935, New York, NY 10016. E-mail: rinberg@nyu.edu. DOI:10.1523/JNEUROSCI.4693-14.2015

Copyright $\odot 2015$ the authors $\quad 0270-6474 / 15 / 3511667-07 \$ 15.00 / 0$
}

and decision making. In olfaction, a single sniff is considered a snapshot of the olfactory world and a unit of perception (Uchida et al., 2006). In fact, rats only need to sample an odor with a single sniff, 125-300 ms, to achieve maximum discrimination accuracy (Uchida and Mainen, 2003; Zariwala et al., 2013). These short sampling times suggest that processing of olfactory information is rapid. Wesson et al. (2008) showed that processing is indeed rapid by demonstrating that the minimal response time with above-chance accuracy from odor inhalation to an action for an innate behavior is $140 \mathrm{~ms}$, which corresponded to the duration of an entire sniff cycle.

In the current work, we sought to define the limits on the speed of olfactory processing. To achieve this, we developed a novel, twoalternative choice, head-fixed odor concentration discrimination paradigm based on temporally precise stimulus delivery.

\section{Materials and Methods}

Animals. Data were collected from six male C57BL/6 mice ( 8 weeks old at the start of training). Mice were on a $12 \mathrm{~h}$ light/dark cycle (lights on at 8:00 pm). All experimental procedures were approved by the Howard Hughes Medical Institute Institutional Animal Care and Use Committee. 
Sniff recording. We implanted a cannula (6 mm long, 25 gauge hypodermic tubing) in the nasal cavity. During experiments, the cannula was connected to a pressure sensor (MPX5050DP; Freescale Semiconductor) with capillary tubing ( $2.4 \mathrm{~mm}$ inner diameter, $7 \mathrm{~cm}$ long) and the pressure signal was sent to an amplifier (Cygnus Technology) with gain set at $50 \times$ and no filters selected. We verified the timing of the pressure signal with a mass flow sensor (AWM 3300; Honeywell), with a typical response time of $1 \mathrm{~ms}$, as described previously (Shusterman et al., 2011). The time delay between the air flow measurement and the pressure measurement did not exceed $4 \mathrm{~ms}$. The sniffing signal was sent to an external microprocessor (Mega1280; Arduino), which waited for the sniffing pressure to cross a threshold value before sending out a digital trigger to the odor valve driver (CoolDrive; NResearch). We set the threshold value to the value of the smallest exhalation peak that we observed at the beginning of each behavioral session. This was sufficient to ensure that, on $>85 \%$ of trials, the odor came on at least $40 \mathrm{~ms}$ before inhalation (see Fig. 2G, inset).

Surgery. Each mouse was implanted with a head bar for head fixation and a sniffing cannula, as described previously (Smear et al., 2011), and allowed to recover for $3 \mathrm{~d}$ before restriction to $1 \mathrm{ml}$ of water per day.

Behavioral setup. See Figure $1 A$ for the behavioral study setup. The odor vial had either $2 \mathrm{ml}$ of amyl acetate (Sigma-Aldrich) or $2 \mathrm{ml}$ of vanillin dissolved in water $(1 \mathrm{~g} / 100 \mathrm{ml})$. The blank vial was either empty or contained water or mineral oil.

We assembled the treadmill using plastic Lego parts: a belt (part \#779938), two wheels (part \#970625), and two axles (part \#970617), and a custom 3D printed main frame. To monitor the treadmill movements, we coupled a DC motor (FK-260SA; Jameco Electronics) to one of the axles of the treadmill. The motor signal was acquired continuously as an analog signal at $1000 \mathrm{~Hz}$ with a National Instruments acquisition board (NI USB-6259). Custom-written MATLAB software (The MathWorks) extracted the data from the acquisition board every $\sim 60 \mathrm{~ms}$, filtered it (fourth-order Butterworth LP filter with a cutoff frequency of $50 \mathrm{~Hz}$, applied bidirectionally, see Fig. $4 A$ for example traces), and then compared it with a threshold value to determine whether a movement had occurred. We used the filter because we occasionally saw voltage spikes in the DC motor signal. We specifically chose the filter to not introduce time shifts or amplitude changes. To verify that the filter did not induce time shifts, we estimated the response time using the unfiltered trace and calculated the difference for each trial between the two response times, which was $0.7 \pm 1.8 \mathrm{~ms}$ (mean \pm absolute mean deviation, all data pooled together). We chose the threshold value of $10 \mathrm{mV}$ to be high enough to disregard the occasional rise in the voltage signal when mice grabbed the belt and lifted it up, which produced a signal of $\sim 5 \mathrm{mV}$. In our offline analysis, we identified movement onset using the continuous motor signal and a lower threshold of $2 \mathrm{mV}$ (see Fig. $4 A$ for the range of peak values; scale bar, $10 \mathrm{~cm} / \mathrm{s}=55 \mathrm{mV}$ ). We occasionally saw changes of direction of movement; however, the choice (and response time) was always scored based on the direction (and onset) of the earliest movement.

To deliver the water reward (1-2 $\mu \mathrm{l})$, we used gravitation flow under the control of a solenoid valve (NResearch). The valve was connected to a lick spout (5 $\mathrm{cm}$ hypodermic tubing, gauge 21 ) via Tygon tubing.

Behavioral training. Training began at least $7 \mathrm{~d}$ after the start of water deprivation. In the first session, mice received water every time they licked. In the second session, mice had to move the treadmill in any direction to receive water. In the third and fourth sessions, we alternated which direction of the treadmill movement was rewarded in blocks of 100 trials to reduce bias for a specific direction. In the next three to five sessions, mice had to move the treadmill leftward/rightward for the low/ high odor concentration. We alternated which concentration was presented in blocks of 100 trials.

In the next eight to nine sessions, the stimuli were in pseudorandom order: in a block of 20 trials, there was an equal number of low and high concentration stimuli in a random order. At this stage, mice typically made errors because either they had a bias for a particular movement direction or they made premature movements before odor inhalation. If in a block of 40 trials mice performed at $<75 \%$ accuracy, we introduced in the next block of 40 trials both of these measures: (1) to reduce the bias, a $90 \%$ probability of repeating a trial if it was an error, and (2) to reduce premature movements, a "grace period" of $200 \mathrm{~ms}$ after the time of odor valve opening, during which any choice made was not considered. The grace period was gradually decreased to $150 \mathrm{~ms}$ and then to $100 \mathrm{~ms}$. The training continued until mice could perform consistently with accuracy $>75 \%$ with no grace period.

For data collection, we used a corrective algorithm: if overall accuracy in the last $55-70$ trials dropped below $70-72 \%$ correct, the next set of 55-70 trials were "corrective trials" and included a grace period of 50$100 \mathrm{~ms}$ and a $90 \%$ probability of repeating an error trial. These corrective trials were not included in the analysis. We included in our analysis all sessions that had at least 100 consecutive trials regardless of their accuracy (no nonconsecutive trials were included). The number of trials per session in our analysis was $315 \pm 140($ mean \pm SD) and the total number of trials per mouse was $478-1226$.

Data analysis. To estimate for each mouse the response time corresponding to $75 \%$ accuracy, we binned the response times using a bin size of $20 \mathrm{~ms}$. We then fitted a logistic function to the choice accuracy as a function of response time using maximum likelihood estimation (MLE) procedures, assuming a binomial distribution of correct and incorrect choices at each time bin (Dobson and Barnett, 2008). In these fits, the probability of being correct is given by the following: $P_{\text {correct }}=(1+\exp$ $\left.\left(b_{0}+b_{1} R T\right)\right)^{-1}$, where $R T$ is the response time. We calculated the SEs for the parameters of the logistic function using the inverse of the information matrix from the MLE fits (Dobson and Barnett, 2008). We then used these SEs to calculate a normal distribution for each parameter. We estimated $95 \%$ confidence intervals for the response time that corresponded to $75 \%$ accuracy by randomly drawing parameter values from their respective distributions 10,000 times.

To create the distribution of peak velocities shown in Figure $4 A$, we excluded values as outliers if they were larger than $\mathrm{q} 3+1.5(\mathrm{q} 3-\mathrm{q} 1)$ or smaller than $\mathrm{q} 1-1.5(\mathrm{q} 3-\mathrm{q} 1)$, where $\mathrm{q} 1$ and $\mathrm{q} 3$ are the 25 th and 75 th percentiles, respectively. This range corresponds to approximately $\pm 2.7 \sigma$ and $99.3 \%$ coverage for normally distributed data.

To compute autocorrelation coefficients, we used the following equation:

$$
C_{k}=\frac{\sum_{i=1}^{N-k}\left(y_{i}-\bar{y}\right)\left(y_{i+k}-\bar{y}\right)}{\sum_{i=1}^{N}\left(y_{i}-\bar{y}\right)^{2}},
$$

where $C_{k}$ is the coefficient at a particular lag $k, N$ is the total number of trials, $y_{i}$ is the value of peak velocity (or odor concentration or direction of movement) for trial $i$, and $\bar{y}$ is the mean value across trials.

Statistical analysis. To evaluate whether a longer time interval from the odor onset to the inhalation onset, $t_{\text {prior }}$, leads to improved accuracy, we calculated the mean, $\mu$, and SE of the distribution shown in Figure $2 \mathrm{H}$ and evaluated the null hypothesis: $\left\{H_{0}: \mu \geq 0\right\}$ using the $t$ test.

To evaluate whether higher peak velocity correlates with higher probability of being correct, we fitted the logistic function: $P_{\text {correct }}=(1+\exp$ $\left(b_{0}+b_{1} v_{\text {peak }}\right)^{-1}$, where $v_{\text {peak }}$ is peak velocity, and then evaluated the null hypothesis: $\left\{H_{0}: b_{1} \leq 0\right\}$. The stated $p$-value is the probability that a single observation from the normal distribution with mean $b_{1}$ and its associated SE will fall in the interval $[-\infty, 0]$.

To investigate whether peak velocity carries additional information about choice accuracy that is not explained by response time, we divided the data into three equal groups according to peak velocity (high, medium, and low peak velocity; see Fig. $4 D$ ) and fitted the logistic function: $P_{\text {correct }}=\left(1+\exp \left(b_{0}+b_{1} R T+b_{2} I+b_{3} I R T+b_{4} J+b_{5} J R T\right)\right)^{-1}$, where $R T$ is the response time and $I$ and $J$ are indicator variables $(I=1$ if the trial is in the medium peak velocity group and zero otherwise and $J=$ 1 if the trial is in the high peak velocity group and zero otherwise). We evaluated the null hypothesis: $\left\{H_{0}: b_{4} \leq 0\right\}$.

\section{Results}

We trained head-fixed mice to discriminate two different concentrations of an odor by moving a horizontal treadmill leftward or rightward with their front limbs (Fig. 1A) in a task similar to 
A

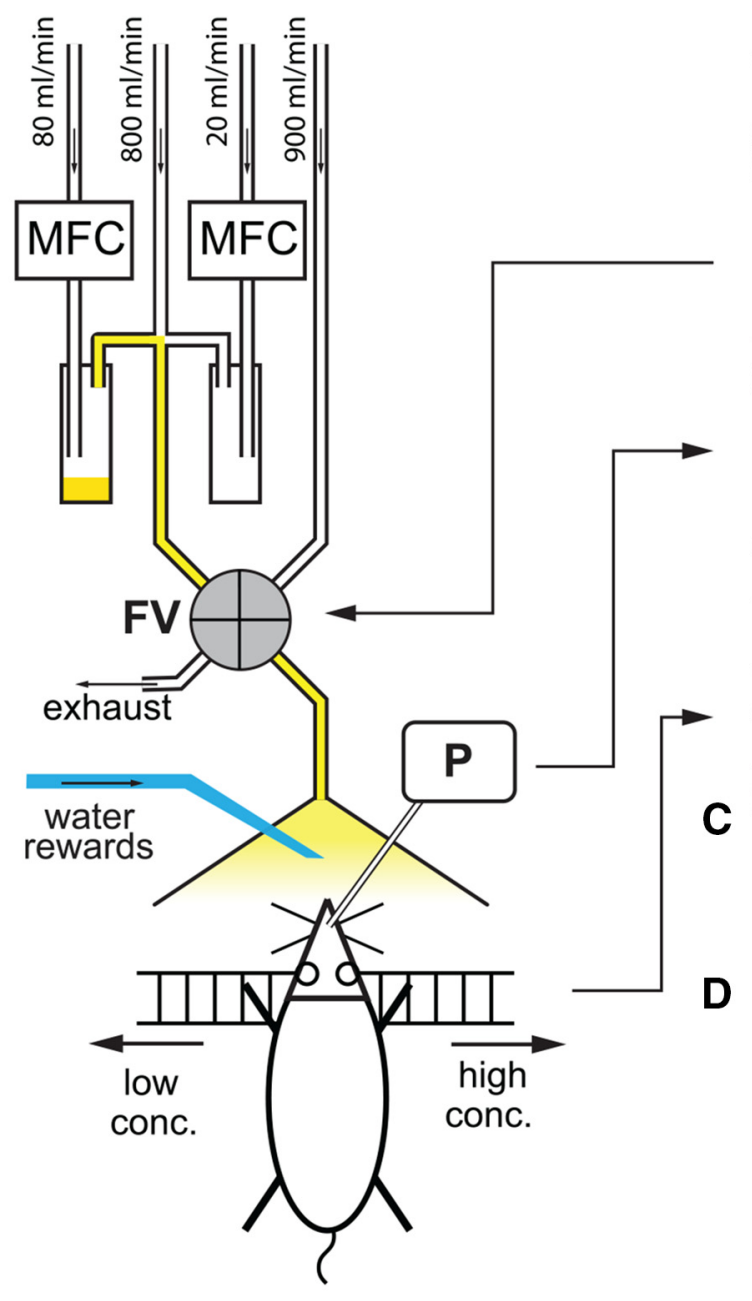

B $\widehat{\dot{j}}$

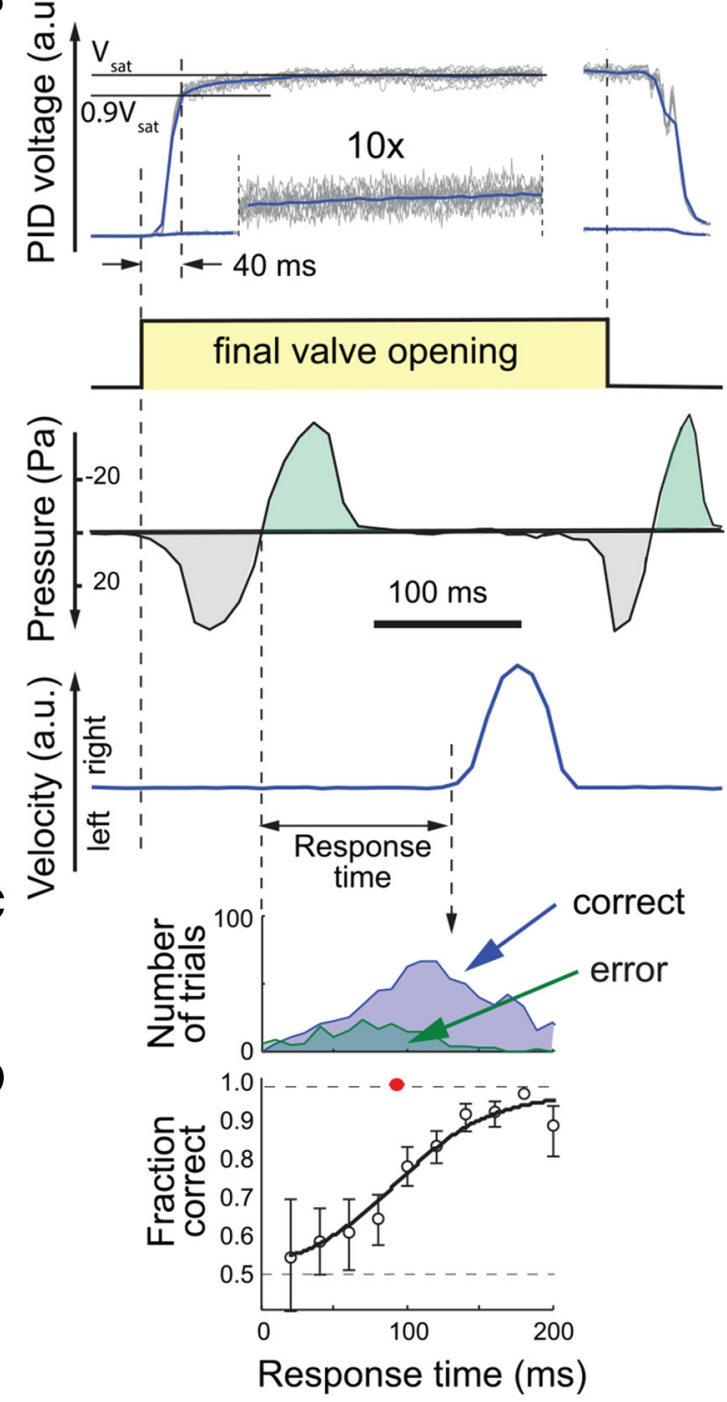

Figure 1. Behavioral task. A, Experimental setup. A head-fixed mouse is positioned in front of the odor port and a water spout with its front paws resting on a treadmill. An implanted sniffing cannula is connected to the pressure transducer (P) to measure the sniffing signal. The mouse is trained to move the treadmill to the left/right in response to the low/high odor concentration stimulus. The odor stimulus is created by combining three parallel gas lines: an air carrier line $(800 \mathrm{ml} / \mathrm{min}$ ) and two nitrogen lines controlled by mass flow controllers (MFCs) (Alicat Scientific) that go through an odor vial (yellow) and a blank vial. For the high concentration stimulus, the odor vial MFC was set to a flow rate of $80 \mathrm{ml} / \mathrm{min}$ and the blank vial MFC was set to a flow rate of $20 \mathrm{ml} / \mathrm{min}$. For the low concentration stimulus, the flow rates were $2 \mathrm{ml} / \mathrm{min}$ and $98 \mathrm{ml} / \mathrm{min}$, respectively. The final valve (FV) (4-way Teflon valve; NResearch) switches the odor line and a blank air line (900 $\mathrm{ml} / \mathrm{min}$ ) between the odor port and the exhaust. $\boldsymbol{B}$, Top, Photoionization detector (PID) (Aurora Scientific) measurement of the time course of the concentration at the odor port for the high and low concentration stimulus (gray lines are individual trials and the blue line is the mean). At $40 \mathrm{~ms}$ after the onset of the final valve, the odor concentration reaches $90 \%$ of its saturated value. Second panel, Time course of the FV opening. Third panel, Pressure measurement of the sniffing signal (example trace). Note that positive pressure is downward. Green/gray area is the inhalation/exhalation interval. Bottom, Limb velocity (example trace). Positive/negative values indicate rightward/leftward motion. The response time is the interval from inhalation onset to movement onset. The delay from movement onset to FV offset was $110 \pm 30 \mathrm{~ms}$ (mean \pm mean absolute deviation). The intertrial interval was $7 \mathrm{~s}$. C, Example distribution of response times for one mouse for correct trials (blue) and incorrect trials (green). D, Response accuracy as a function of response time ( $20 \mathrm{~ms}$ time bins). Error bars are $95 \%$ confidence intervals. The solid line is a logistic fit to the data. The red filled circle is the time that corresponds to $75 \%$ accuracy and the error bar through it is the $95 \%$ confidence interval.

that of Sanders and Kepecs (2012). The key feature of our setup was precise triggering of the odor stimulus delivery during exhalation so that the odor stimulus stabilized before the first stimulus inhalation, thus increasing the trial to trial reproducibility (Fig. $1 B)$. To ensure that the behavioral task was not limited by stimulus noise, we chose two stimuli that were clearly distinguishable from each other $(0.02 \%$ and $0.8 \%$ of saturated vapor pressure of amyl acetate). The temporal precision of the stimulus delivery system was higher than the temporal resolution of the olfactory system, $\sim 10 \mathrm{~ms}$, as reported in prior work (Smear et al., 2011).

Under these conditions, mice responded very rapidly. Their median response time, defined as the time from inhalation onset to movement onset, varied from 60 to $130 \mathrm{~ms}$ (Figs. 1C, 2A). Their choice accuracy increased for longer response times and this functional relationship was well fit by a logistic function $\left(R^{2}\right.$ $=0.89-0.98$ across mice; Figs. $1 D, 2 B$ ). Based on these fits, mice achieved $75 \%$ accuracy for movements initiated $70-90 \mathrm{~ms}$ after the onset of inhalation (Fig. 2B), which was before a single sniff was over (Fig. 2C,D), and achieved $95 \%$ accuracy for movements initiated $100-150 \mathrm{~ms}$ after the onset of inhalation. Eliminating odor cues by having the same odor concentration in both vials decreased the choice accuracy to chance levels ( $p>0.23$, binomial test; Fig. $2 E$ ), thus showing that mice relied on odor information to solve the task. These experiments show that mice can 

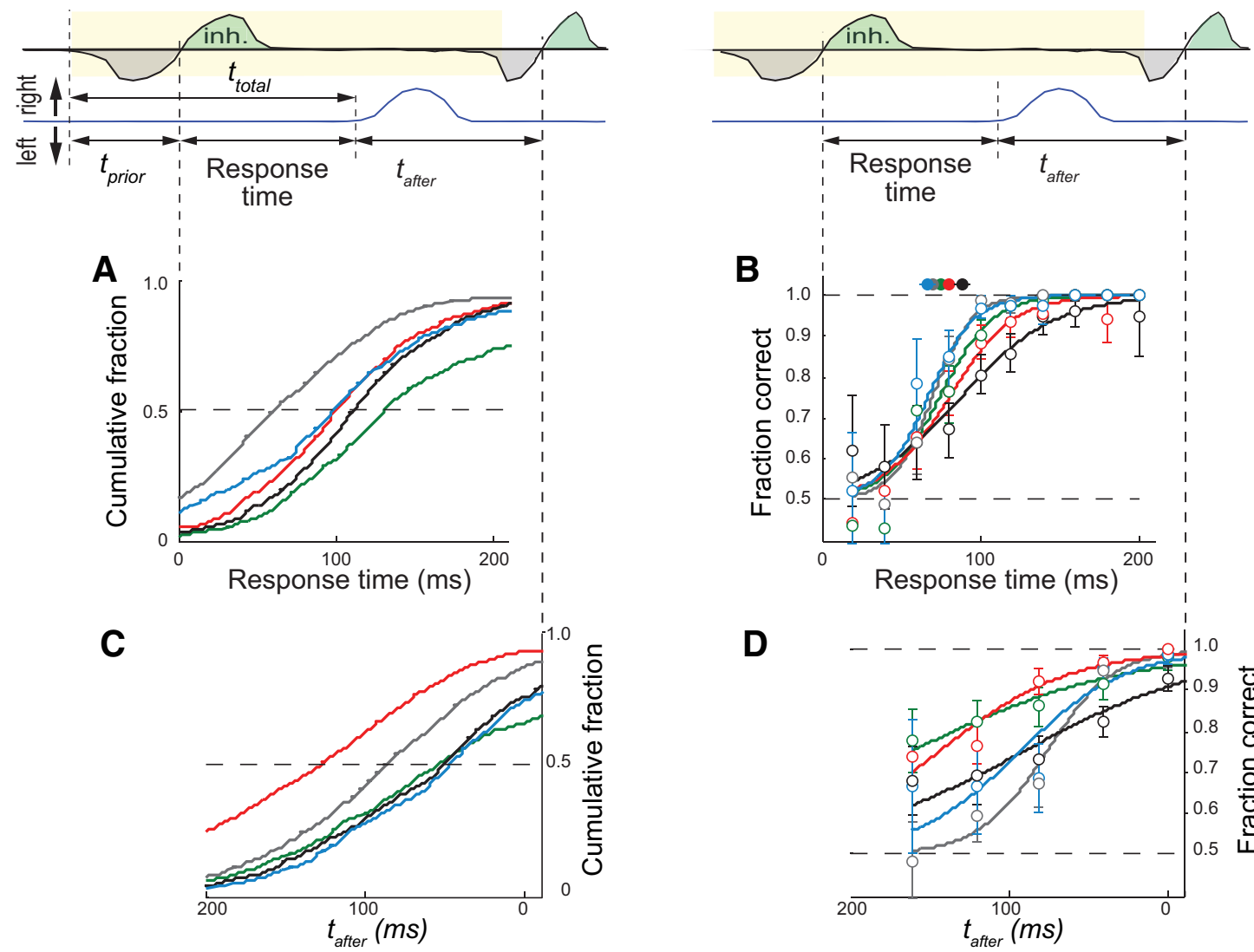

B

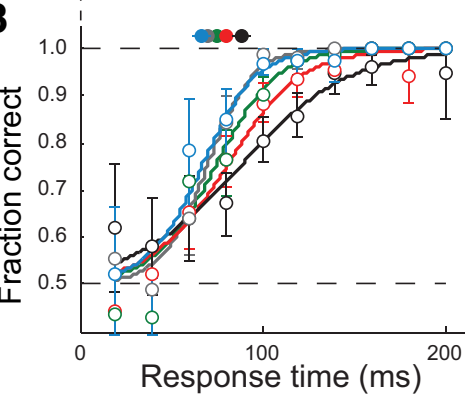

D
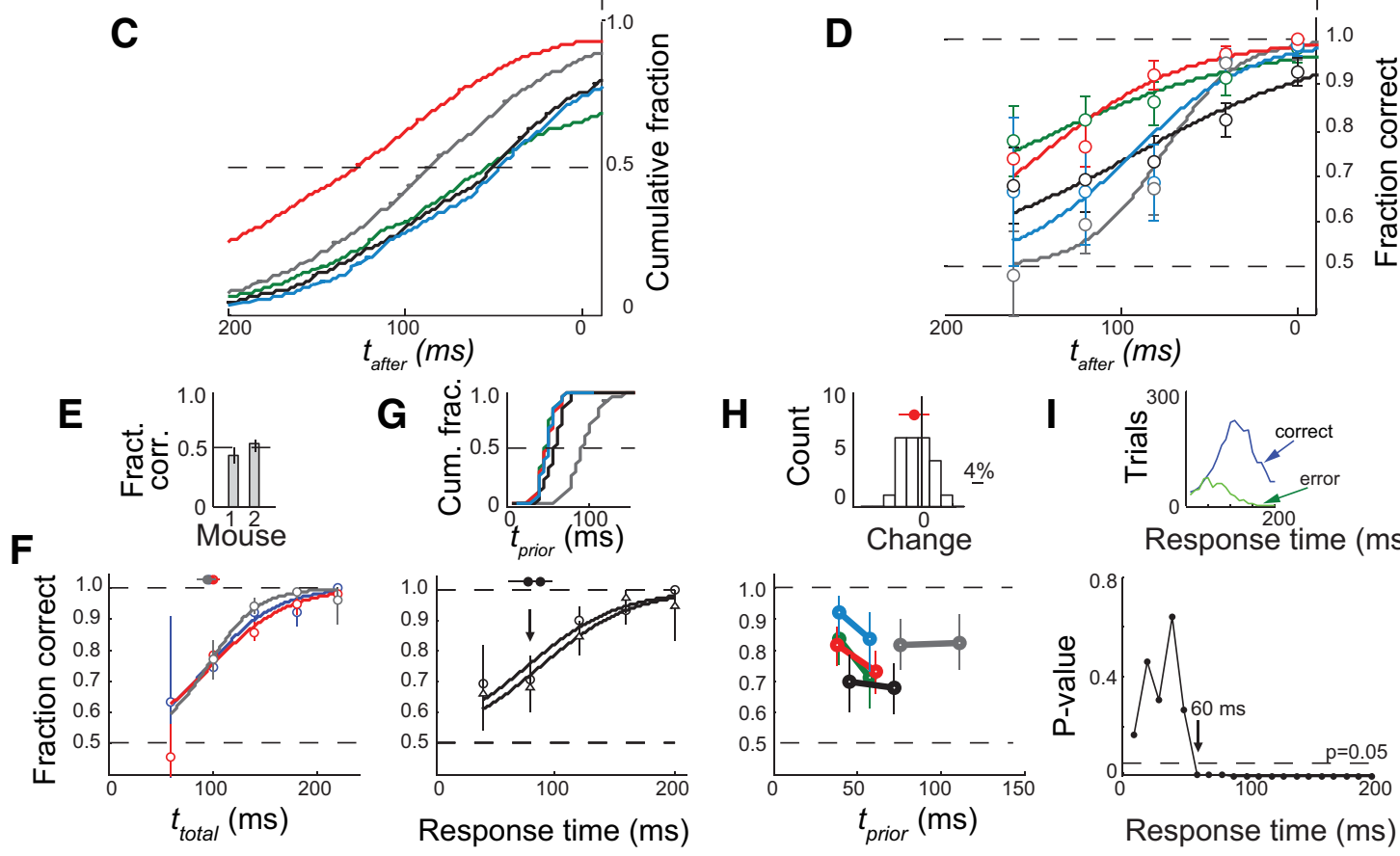

Response time (ms)

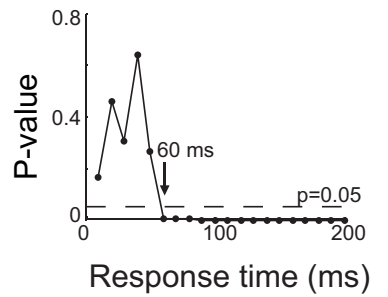

Figure 2. Rapid olfactory decisions. $\boldsymbol{A}$, Cumulative distributions of response times from all trials for five mice. Each mouse is indicated by a different color. $\boldsymbol{B}$, Response accuracy as a function of response time. Response times are binned in $20 \mathrm{~ms}$ bins (the $60 \mathrm{~ms}$ bin corresponds to response times that are $50-70 \mathrm{~ms}$ long). Error bars are $95 \%$ confidence intervals. The solid lines are logistic fits to the data. The filled circles are the times that correspond to $75 \%$ accuracy for each mouse and the error bars through them are the $95 \%$ confidence intervals. C, Cumulative distributions of $t_{\text {after }}$ : the interval from movement initiation to the onset of the next sniff. $\boldsymbol{D}$, Response accuracy as a function of $t_{\text {after }}$. All mice reached $75 \%$ accuracy before the end of the first sniff. $\boldsymbol{E}$, Response accuracy for two mice in a control experiment in which both vials had the same odor concentration. $\boldsymbol{F}$, Response accuracy as a function of $t_{\text {total }}$, the interval from the time of the trigger to movement onset, for three mice. In this experiment, odor delivery was triggered $10-25$ ms before inhalation (range of median times across mice). $G$, Response accuracy as a function of response time for different values of $t_{\text {prior }}$, the interval from the time of the trigger to inhalation onset. Data are from one mouse as an example. Trials were divided into three equal groups according to values of $t_{\text {prior: }}$ circles/triangles are the data for the first/last third of the $t_{\text {prior }}$ distribution. Top insert, Cumulative distribution of $t_{\text {prior }}$ for five mice. $\boldsymbol{H}$, Response accuracy for the first and the last third of the $t_{\text {prior }}$ distribution for response times between 60 and $100 \mathrm{~ms}$ (see arrow in $\boldsymbol{G}$ ) for five mice. The values for $t_{\text {prior }}$ are the medians for each group. Top insert, Distribution of the pairwise difference in accuracy between the long $t_{\text {prior }}$ group and the short $t_{\text {prior }}$ group for each individual response time bin in $G$. Data are pooled across mice. Red circle and red line are the distribution mean and SE, respectively. $I$, Top insert, Distribution of response times ( $10 \mathrm{~ms}$ bins) for correct trials (blue) and incorrect trials (green) for all mice in $\boldsymbol{A}$. The $p$-value is the probability of observing the number of correct trials in each time bin assuming a binomial distribution of correct and incorrect trials and a performance accuracy of $50 \%$.

make discriminations of different odor concentrations in tens of milliseconds.

Our estimation of response times is based on the assumption that mice need to inhale the odor to make a decision. If mice can acquire odor information during exhalation, then our response time estimates would be extended by $50-90 \mathrm{~ms}$ (range of median times across mice), which is the interval from the onset of odor delivery to the onset of odor inhalation, $t_{\text {prior }}$ (Fig. $2 G$, top). To exclude this possibility, we triggered odor delivery $10-25 \mathrm{~ms}$ before inhalation (median $t_{\text {prior }}$ across mice). We found that responses initiated $100 \mathrm{~ms}$ after odor onset had an accuracy of $\sim 75 \%$ (Fig. $2 F$ ). Given that the odor reaches the odor port $\sim 25$ $\mathrm{ms}$ after final valve onset (Fig. $1 B$ ), mice can use up to $75 \mathrm{~ms}$ to reach $75 \%$ accuracy. This is in agreement with response times in the main experiment, in which mice reached $75 \%$ accuracy 70-90 ms after the onset of odor inhalation. Furthermore, we 


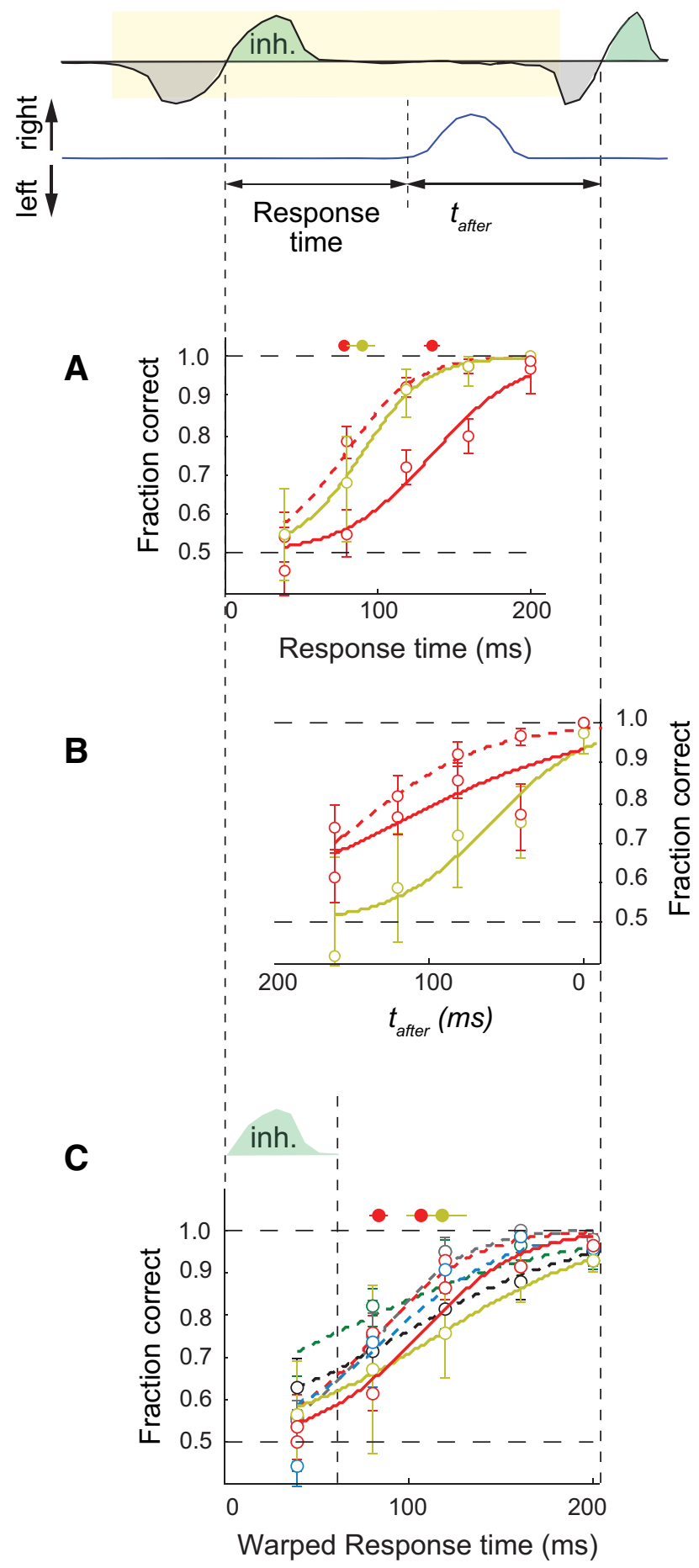

Figure 3. Rapid discriminations for the nontrigeminal stimulant vanillin. $\boldsymbol{A}$, Response accuracy as a function of response time for the odors vanillin (solid lines) and amyl acetate (dashed line) for two mice. $\boldsymbol{B}$, Response accuracy as a function of $t_{\text {after }}$ for vanillin (solid lines) and amyl acetate (dashed line) for two mice. $\boldsymbol{C}$, Response accuracy as a function of response time in sniff phase coordinates for vanillin (solid lines) and amyl acetate (dashed lines) for all mice in $\boldsymbol{A}$ and in Figure $2 B$. All inhalation/exhalation intervals were stretched or compressed so that they were the length of the average inhalation/exhalation interval computed from all mice. The warped response time is the response time stretched/compressed with its corresponding sniff interval.

divided the data in the main experiment into three groups depending on $t_{\text {prior. }}$. We found that choices with similar response times after the onset of inhalation were not significantly more accurate if they were preceded by a longer odor interval before inhalation ( $p=0.99$, see Materials and Methods; Fig. 2G-H).
Finally, to assess whether a bout of rapid sniffs resulted in shorter response times because there may have been some odor inhaled during $t_{\text {prior }}$, we divided the data in the main experiment into two groups: the $25 \%$ of trials with the shortest prior intersniff interval and the remainder of the trials. We found that shorter prior intersniff intervals were not followed by significantly shorter response times in $3 / 4$ of mice ( $p=0.06-0.93$, Wilcoxon rank-sum test; we excluded one mouse because we did not acquire its full prior sniff). Therefore, we found no evidence that mice used odor information before inhalation.

To address whether the trigeminal pathway is involved in these rapid discriminations, we trained two mice to discriminate two concentrations of vanillin, an odorant that does not activate the trigeminal (Doty et al., 1978; Frasnelli et al., 2011). Some of the trigeminal neurons in the nasal epithelium have direct projections to the brainstem (Schaefer et al., 2002), so they create a direct and potentially rapid pathway for processing of odor information. For this odorant, mice reached $75 \%$ discrimination accuracy for responses initiated $90-140 \mathrm{~ms}$ after inhalation onset (Fig. 3A), which is about $50 \mathrm{~ms}$ longer than the response times for amyl acetate (Fig. $3 A$ ); however, this time is still shorter than the duration of the sniff cycle (Fig. $3 B$ ). This shift may also be attributed to a significantly lower absolute concentration of vanillin, which was $10^{5}$ lower than that of amyl acetate. To further compare our results for amyl acetate and vanillin, we computed the response time in sniff phase coordinates or warped time coordinates using procedures proposed previously by Shusterman et al. (2011). Briefly, we stretched or compressed individual sniffs so that each inhalation/exhalation was the length of the average inhalation/exhalation computed from all mice; the response time was stretched/compressed with its corresponding interval. In this warped sniff time, we found that the shift between amyl acetate and vanillin was only about $20 \mathrm{~ms}$ (Fig. 3C), consistent with the observation that mice take longer sniffs for vanillin $(p<0.05$, Wilcoxon rank-sum test). Overall, this experiment shows that mice can process odor information rapidly, even in the absence of trigeminal stimulation.

To further understand the processing of odor information at these short timescales, we quantified the peak movement velocity (Fig. 4A), which can be thought of as the vigor of the limb movement. Does the behavioral action simply relay the outcome of these rapid decisions or does it also carry information about the likelihood that the decision is correct? We found that the higher the peak velocity, the more likely it was that the choice was correct $(p<0.01$; Fig. $4 B)$. For this analysis, we excluded all the trials in which peak velocity occurred after the mice had received feedback on their choice $(8-16 \%$ of the trials across mice; similar results were obtained with all trials included). The correlation between peak velocity and accuracy may be explained by intertrial correlation in performance. A mouse may perform with higher accuracy and with stronger limb movements during one block of trials and then decrease its accuracy and peak velocity in another block, for example, due to changes in motivation. However, this possibility cannot explain the results for the four of five mice for which the different values for peak velocity were largely intermixed across trials, as shown by the autocorrelation coefficients for the trial-to-trial sequence of peak velocities (Fig. 4C). Finally, we have shown that accuracy covaries with both response time and peak movement velocity. Peak movement velocity, however, does not simply reflect the same accuracy information as response time, because movements initiated at the same time are more likely to be correct if their peak velocity is higher $(p<$ 
A

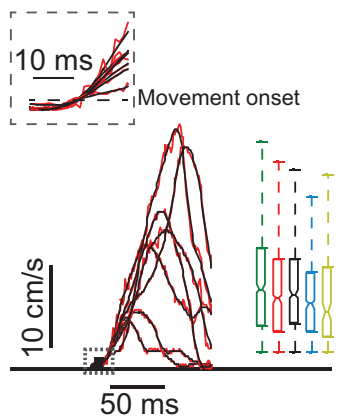

B

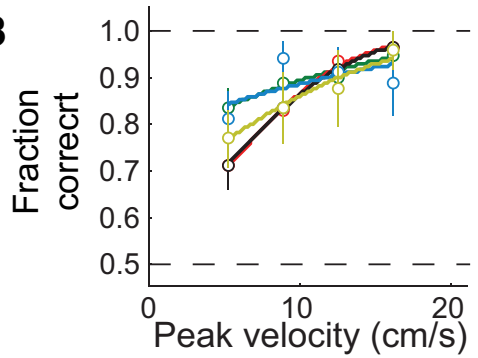

C
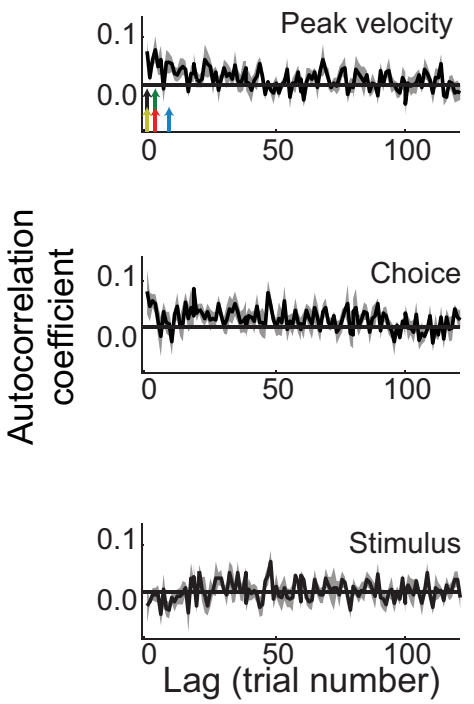

D
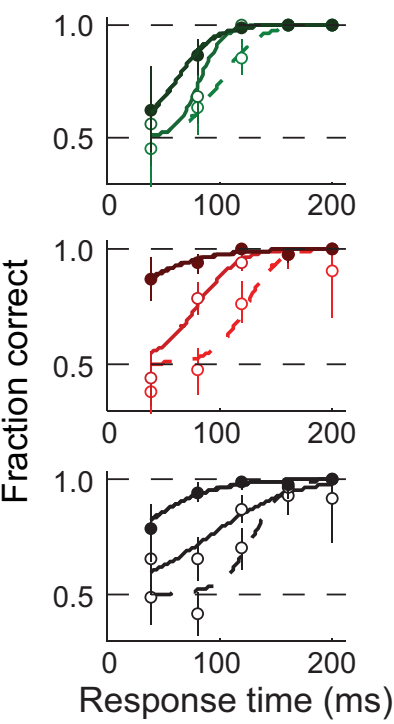

Figure 4. Correct choices have higher peak velocities than incorrect choices. $\boldsymbol{A}$, Left, Example velocity traces from seven individual trials (red is unfiltered, black is filtered). Dashed area is magnified at the top. Right, Peak velocity distributions for 5 mice. Here and throughout, different colors indicate different mice, the same as in Figures 2 and 3 . Each boxplot shows the median, the $25^{\text {th }}$ and $75^{\text {th }}$ percentiles, and the lowest and highest values in the distribution that were not outliers (two to 10 trials were excluded across mice as outliers). $\boldsymbol{B}$, Response accuracy as a function of peak velocity for five mice. Solid lines are logistic fits to the data. Error bars are $95 \%$ confidence intervals. C, Autocorrelation coefficient averaged across mice (solid line is the mean, gray area is the SEM) for the trial-to-trial sequence of peak velocities (top), choice (rightward or leftward movement; middle), and stimulus (high or low concentration; bottom). Arrows indicate the first trial number for each mouse when three consecutive coefficients, computed from the sequence of peak velocities, are not significantly different from coefficients computed from a randomly shuffled sequence 10,000 times $(p>0.05)$. D , Response accuracy as a function of response time for different peak velocities for the three mice that had a sufficient number of trials for this analysis. For each mouse, data were divided into three equal groups: low peak velocity (dashed line), medium peak velocity (light solid line), and high peak velocity (dark solid line). Error bars are $95 \%$ confidence intervals. Lines are logistic fits to the data.

0.05; Fig. $4 D$; we excluded two mice that had too few trials because this analysis requires a large number of trials).

\section{Discussion}

Together, this study shows that mice can make olfactory discriminations of 75\% accuracy in 70-90 ms after odor inhalation. For a low concentration and nontrigeminal odorant, this time was $90-140 \mathrm{~ms}$, showing that processing of olfactory information is rapid even in the absence of trigeminal stimulation.

Further, this study shows that the triggered behavioral action does not simply relay the outcome of the decision, but it also carries information about the likelihood that the decision is correct, consistent with previous work in humans in which arm movements were used as a motor response (Selen et al., 2012). What might peak movement velocity indicate? We speculate that high peak movement velocity is indicative of a "prepared" motor system to act and is therefore indicative of shorter motor delays, perhaps similar to the expression of saccades in primates (Fischer and Boch, 1983). It is known that the response time is made up of both a decision time, which improves accuracy, and a nondecision time, which reflects sensory and motor delays (Resulaj et al., 2009). We suspect that movements with higher peak velocity may have shorter nondecision times and thus may be more likely to reflect longer decision times and hence higher accuracies.

Our study sought to define the limit on the speed of olfactory processing. Ultimately, the distribution of response times chosen by the animal depends on weighing many factors, such as the cost of a wrong choice (for example punishment), the value of a reward now versus a surer reward later, the cost of waiting for another trial (i.e., the intertrial period), etc. Although the response time associated with 75\% accuracy in our task was 70-90 ms for amyl acetate, the median response time across mice was $60-130 \mathrm{~ms}$.

Previous work has reported that innate (nonlearned) responses about the novelty of a stimulus can be made by rodents in as little as $140 \mathrm{~ms}$ (for amyl acetate among other odors; Wesson et al., 2008). This is the minimal response time, which is the earliest response time with above chance accuracy reported for odor detection or discrimination by previous studies. In our current work, the minimal response time for concentration discrimination was $60 \mathrm{~ms}$ (Fig. 2I). One explanation for this difference is that we used limb movements to indicate choice, whereas the study by Wesson et al. (2008) used the increase in sniffing rate in response to novel odors to measure choice. Specifically, rats initiate a second sniff faster when the odor is novel compared with when the odor is a learned one, so the minimum duration of the first sniff for novel odors can be used as a measure of response time. However, this measure of response time depends on how fast rats sniff. In contrast, limb movements are decoupled from sniffing and can be initiated at any time.

Response times of 70-90 ms (mean $76 \mathrm{~ms}$ ) are very short considering that the first odor responses in sensory areas are detected at $\sim 30 \mathrm{~ms}$ after odor inhalation (Cury and Uchida, 2010). Further, motor delays for limb movements in mice after optogenetic stimulation of motor cortex are $\sim 25 \mathrm{~ms}$ (Hira et al., 2009). Therefore, sensory and motor delays could amount to $\sim 55 \mathrm{~ms}$, leaving as little as $\sim 20 \mathrm{~ms}$ for processing. This window puts a strong limit on the underlying neural computations and it implies that only the first action potentials across a population of neurons encode this sensory information.

This estimate of processing time is also short for the nontrigeminal odorant: response times are 90-140 ms (mean $115 \mathrm{~ms}$ ), 
leaving $\sim 60 \mathrm{~ms}$ for sensory processing. We expect that this is an upper bound for the processing time of nontrigeminal odorants because vanillin has a poor solubility and thus a low concentration $\left(10^{5}\right.$ lower than that of amyl acetate), which presumably results in a longer delay from odor inhalation to activation of the olfactory receptors.

Previous studies have reported a processing time of as little as $50 \mathrm{~ms}$ for mixed trigeminal and olfactory odorants (amyl acetate among other odorants; Wesson et al., 2008). This time is estimated by subtracting the time of the earliest responding glomerulus, which is detected at $120 \pm 45 \mathrm{~ms}$ after odor inhalation (mean $\pm \mathrm{SD}$ ), from the response time in the same trials. However, a key distinction is that mice in our task frequently responded before the earliest responding glomeruli reported by Wesson et al. (2008): the median response time in our task was 60-130 ms. It remains unclear what contributes to this difference, but the explanation will likely necessitate neural recordings.

Future studies that combine this behavior with neural recordings will elucidate the neural basis of these decisions. In sensory areas, olfactory information can be read out in the sequence of action potentials that tile the entire sniff cycle (Cury and Uchida, 2010; Shusterman et al., 2011). This temporal arrangement may allow discrimination of two different stimuli early in the sniff cycle-that is, within a few action potentials. Indeed, our study suggests that the relevant action potentials forming the neural basis of these decisions are fired in just a few tens of milliseconds.

\section{References}

Cury KM, Uchida N (2010) Robust odor coding via inhalation-coupled transient activity in the mammalian olfactory bulb. Neuron 68:570-585. CrossRef Medline

Dobson AJ, Barnett AG (2008) An introduction to generalized linear models. London: Chapman \& Hall.

Doty RL, Brugger WE, Jurs PC, Orndorff MA, Snyder PJ, Lowry LD (1978) Intranasal trigeminal stimulation from odorous volatiles: psychometric responses from anosmic and normal humans. Physiol Behav 20:175-185. CrossRef Medline

Fischer B, Boch R (1983) Saccadic eye movements after extremely short reaction times in the monkey. Brain Res 260:21-26. CrossRef Medline

Frasnelli J, Hummel T, Berg J, Huang G, Doty RL (2011) Intranasal localizability of odorants: influence of stimulus volume. Chem Senses 36:405410. CrossRef Medline

Hira R, Honkura N, Noguchi J, Maruyama Y, Augustine GJ, Kasai H, Matsuzaki M (2009) Transcranial optogenetic stimulation for functional mapping of the motor cortex. J Neurosci Methods 179:258-263. CrossRef Medline

Resulaj A, Kiani R, Wolpert DM, Shadlen MN (2009) Changes of mind in decision-making. Nature 461:263-266. CrossRef Medline

Sanders JI, Kepecs A (2012) Choice Ball: a response interface for psychometric discrimination in head-fixed mice. J Neurophysiol 108:34163423. CrossRef Medline

Schaefer ML, Böttger B, Silver WL, Finger TE (2002) Trigeminal collaterals in the nasal epithelium and olfactory bulb: a potential route for direct modulation of olfactory information by trigeminal stimuli. J Comp Neurol 444:221-226. CrossRef Medline

Selen LP, Shadlen MN, Wolpert DM (2012) Deliberation in the motor system: reflex gains track evolving evidence leading to a decision. J Neurosci 32:2276-2286. CrossRef Medline

Shusterman R, Smear MC, Koulakov AA, Rinberg D (2011) Precise olfactory responses tile the sniff cycle. Nat Neurosci 14:1039-1044. CrossRef Medline

Smear M, Shusterman R, O’Connor R, Bozza T, Rinberg D (2011) Perception of sniff phase in mouse olfaction. Nature 479:397-400. CrossRef Medline

Uchida N, Mainen ZF (2003) Speed and accuracy of olfactory discrimination in the rat. Nat Neurosci 6:1224-1229. CrossRef Medline

Uchida N, Kepecs A, Mainen ZF (2006) Seeing at a glance, smelling in a whiff: limits on temporal integration in perception. Nat Rev Neurosci 7:485-491. CrossRef Medline

Wesson DW, Carey RM, Verhagen JV, Wachowiak M (2008) Rapid encoding and perception of novel odors in the rat. PLoS Biol 6:e82. CrossRef Medline

Zariwala HA, Kepecs A, Uchida N, Hirokawa J, Mainen ZF (2013) The limits of deliberation in a perceptual decision task. Neuron 78:339-351. CrossRef Medline 